\title{
Trying to understand the in-plane/out-of-plane effect in long-range correlations*
}

\author{
Yogiro Hama ${ }^{\dagger}$ \\ Universidade de São Paulo, \\ E-mail: hama@fma.if.usp.br
}

Rone P.G. Andrade

Universidade de São Paulo,

E-mail: randradedif.usp.br

\section{Frédérique Grassi}

Universidade de São Paulo,

E-mail: grassi@fma.if.usp

\section{Wei-Liang Qian}

Universidade Federal de Ouro Preto

E-mail: weiliang.qian@gmail.com

\begin{abstract}
We show that the NexSPheRIO code qualitatively reproduces the in-plane/out-of-plane effect, observed in the long-range two-particle correlation in Au+Au collisions at $200 \mathrm{~A} \mathrm{GeV}$. The origin of the effect is clarified with the use of a simplified model, with one peripheral high-energydensity tube in a smooth background, with boost-invariant longitudinal expansion.
\end{abstract}

The Seventh Workshop on Particle Correlations and Femtoscopy

September 20 - 242011

University of Tokyo, Japan

${ }^{*}$ The main content of this paper appears also in our ISMD2011 Proceedings manuscript, entitled "On the Origin of the Trigger-Angle Dependence of the Ridge Structure" (to appear in Prog.Theor.Phys.Suppl.) [1].

† Speaker. 


\section{Introduction}

One of the most striking results in relativistic heavy-ion collisions at RHIC and LHC, is the existence of structures in the two-particle correlations [2, 3, 4, 5, 6, 7] plotted as function of the pseudorapidity difference $\Delta \eta$ and the angular spacing $\Delta \phi$. The so-called ridge has a narrow $\Delta \phi$ located around zero and a long $\Delta \eta$ extent. The other structure located opposite has a single or double hump in $\Delta \phi$.

In a previous work,[8] we got the ridge structure in a purely hydrodynamic model. What is essential to producing ridges in hydrodynamic approach are: i) Event-by-event fluctuating initial conditions (IC); and besides, ii) Very bumpy tubular structure in the IC. Our code NeXSPheRIO uses this kind of IC, produced by NEXUS event generator,[9] connected to SPheRIO hydro code.[10] In our previous studies on ridge, by using 3D NeXSPheRIO code, we obtained some of the experimentally known properties such as

- centrality dependence, $[11,12,13]$

- trigger-direction dependence in non-central windows,[11, 12, 13, 14]

- $p_{T}$ dependence, $[12,13]$

However, what is the origin of ridges? In order to understand the dynamics of ridge formation, we studied carefully what happens in the neighborhood of a peripheral high-energy tube, introducing what we call boost-invariant one-tube model.[11]

\section{In-plane/out-of-plane effect}

In non-central collisions (20-60\% centrality), data have been obtained, fixing the azimuthal angle of the trigger $\left(\phi_{S}\right)$ with respect to the event plane. $[16,17]$ As shown in Fig. 1, the ridge structure in $\Delta \phi$ depends on the trigger direction, especially on the away-side. It changes continuously from one-broad-peak structure at $\phi_{S}=0$ (in-plane trigger), to double-peak structure at $\phi_{S}=\pi / 2$ (out-of-plane trigger). The change is particularly manifest for larger transverse momentum of the associated particle. We tried to see whether this behavior can be obtained with our NeXSPheRIO code.

The results are shown in Fig. 1, with solid lines. Here, we emphasize that these are results of no-parameter computations, having been all the parameters fitted previously using the singleparticle $\eta$ and $p_{T}$ distributions. So, we think NeXSPheRIO code can reproduce the data quite reasonably.

Now, how is this effect produced? Trying to clarify the origin of the effect, we again used the boost-invariant one-tube model, now adapted to non-central collisions. In Ref. [18], by considering the (20-30)\% centrality window, we took as the background the average energy-density distribution obtained with NeXSPheRIO, which now has an elliptical shape. A randomly distributed peripheral tube is put on top of this as our initial conditions. As shown there, this model does reproduce the in-plane/out-of-plane effect, as exhibited by two-particle correlation data. ${ }^{1}$ Here, we reproduce in

\footnotetext{
${ }^{1}$ See more details in Ref. [18].
} 


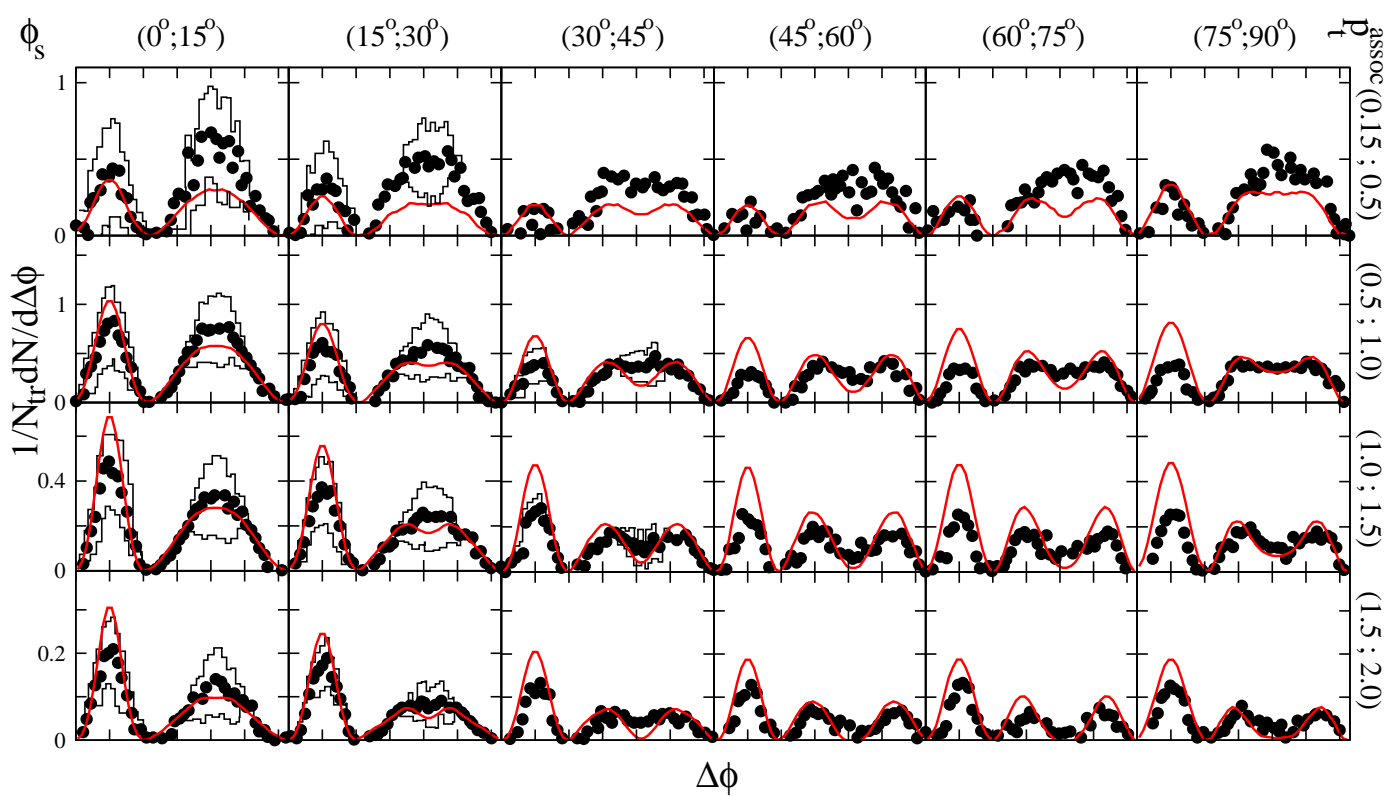

Figure 1: Comparison of NeXSPheRIO results (solid curves) on the trigger-angle dependence of twoparticle correlation, as function of $\Delta \phi$, with data points [16].

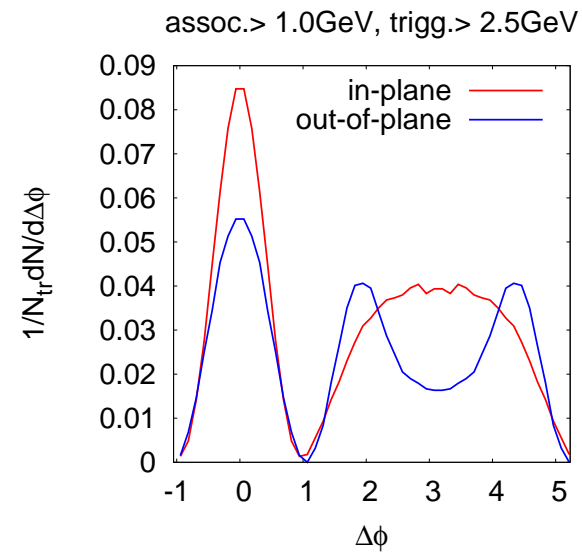

Fig. 2 only the final results, after integration over the random tube position, for two different trigger angles $\left(\phi_{S} \simeq 0\right.$ : in-plane, and $\phi_{S} \simeq \pi / 2$ : out-of-plane) .

However, the mechanism of this effect is still not transparent. In order to make it clearer, we try the following analytical parametrization of the angular distribution, which is valid if the amplitude of fluctuations is small enough:

Figure 2: Two-particle correlation as function of $\Delta \phi$ for two different trigger angles.

$$
\frac{d N}{d \phi}\left(\phi, \phi_{t}\right)=\frac{d N_{b g d}}{d \phi}(\phi)+\frac{d N_{\text {tube }}}{d \phi}\left(\phi, \phi_{t}\right)
$$

where

$$
\begin{aligned}
\frac{d N_{\text {bgd }}}{d \phi}(\phi) & =\frac{N_{b}}{2 \pi}\left(1+2 v_{2}^{b} \cos (2 \phi)\right) \quad \text { and } \\
\frac{d N_{\text {tube }}}{d \phi}\left(\phi, \phi_{t}\right) & =\frac{N_{t}}{2 \pi} \sum_{n=2,3} 2 v_{n}^{t} \cos \left(n\left[\phi-\phi_{t}\right]\right) .
\end{aligned}
$$

The azimuthal angle $\phi$ is measured with respect to the event plane, and $\phi_{t}$ is the location of the randomly distributed tube. For simplicity, in the tube contribution, Eq.(2.3), we retained only 
the dominant $v_{2}^{t}$ and $v_{3}^{t}$ terms, although in a more precise hydro calculation higher-order Fourier components do appear. They are small.

The two-particle correlation is given by

$$
\left\langle\frac{d N_{\text {pair }}}{d \Delta \phi}\left(\phi_{s}\right)\right\rangle=\left\langle\frac{d N_{\text {pair }}}{d \Delta \phi}\left(\phi_{s}\right)\right\rangle^{\text {proper }}-\left\langle\frac{d N_{\text {pair }}}{d \Delta \phi}\left(\phi_{s}\right)\right\rangle^{\text {mixed }}
$$

where, in one-tube model,

$$
\begin{aligned}
\left\langle\frac{d N_{\text {pair }}}{d \Delta \phi}\right\rangle^{\text {proper }} & =\int \frac{d \phi_{t}}{2 \pi} f\left(\phi_{t}\right) \frac{d N}{d \phi}\left(\phi_{s}, \phi_{t}\right) \frac{d N}{d \phi}\left(\phi_{s}+\Delta \phi, \phi_{t}\right) \quad \text { and } \\
\left\langle\frac{d N_{\text {pair }}}{d \Delta \phi}\right\rangle^{\text {mixed }} & =\int \frac{d \phi_{t}}{2 \pi} f\left(\phi_{t}\right) \int \frac{d \phi_{t}^{\prime}}{2 \pi} f\left(\phi_{t}^{\prime}\right) \frac{d N}{d \phi}\left(\phi_{s}, \phi_{t}\right) \frac{d N}{d \phi}\left(\phi_{s}+\Delta \phi, \phi_{t}^{\prime}\right) .
\end{aligned}
$$

Here, $\phi_{s}$ is the trigger angle ( $\phi_{s}=0$ for in-plane and $\phi_{s}=\frac{\pi}{2}$ for out-of-plane trigger) and $f\left(\phi_{t}\right)$ is the distribution function of the tube. We will take $f\left(\phi_{t}\right)=1$, for simplicity. Notice that in the mixed events, integrations both over $\phi_{t}$ and $\phi_{t}^{\prime}$ are required, whereas only one integration, over $\phi_{t}$ is enough for proper events in the averaging procedure. This difference becomes essential.

Using our simplified parametrization, Eqs.(2.1), (2.2), (2.3) and, by averaging over events, the two-particle correlation for the in-plane trigger is given as

$$
\left\langle\frac{d N_{\text {pair }}}{d \Delta \phi}\right\rangle_{\text {in }}^{\text {proper }}=\frac{<N_{b}^{2}>}{(2 \pi)^{2}}\left(1+2 v_{2}^{b}\right)\left(1+2 v_{2}^{b} \cos (2 \Delta \phi)\right)+\left(\frac{N_{t}}{2 \pi}\right)^{2} \sum_{n=2,3} 2\left(v_{n}^{t}\right)^{2} \cos (n \Delta \phi)
$$

and

$$
\left\langle\frac{d N_{\text {pair }}}{d \Delta \phi}\right\rangle_{\text {in }}^{\text {mixed }}=\frac{<N_{b}>^{2}}{(2 \pi)^{2}}\left(1+2 v_{2}^{b}\right)\left(1+2 v_{2}^{b} \cos (2 \Delta \phi)\right) .
$$

Observe the difference between the factors multiplying the background terms of the proper- and the mixed-event correlations. So, by subtracting Eq.(2.8) from Eq.(2.7), the resultant in-plane correlation is

$$
\begin{aligned}
\left\langle\frac{d N_{\text {pair }}}{d \Delta \phi}\right\rangle_{\text {in-plane }} & =\frac{<N_{b}^{2}>-<N_{b}>^{2}}{(2 \pi)^{2}}\left(1+2 v_{2}^{b}\right)\left(1+2 v_{2}^{b} \cos (2 \Delta \phi)\right) \\
& +\left(\frac{N_{t}}{2 \pi}\right)^{2} \sum_{n=2,3} 2\left(v_{n}^{t}\right)^{2} \cos (n \Delta \phi)
\end{aligned}
$$

i.e., if the multiplicity fluctuates the background elliptic flow does contribute to the correlation.

Similarly, the out-of-plane correlation is given as

$$
\begin{aligned}
\left\langle\frac{d N_{\text {pair }}}{d \Delta \phi}\right\rangle_{\text {out-of-plane }} & =\frac{\left\langle N_{b}^{2}>-<N_{b}>^{2}\right.}{(2 \pi)^{2}}\left(1-2 v_{2}^{b}\right)\left(1-2 v_{2}^{b} \cos (2 \Delta \phi)\right) \\
& +\left(\frac{N_{t}}{2 \pi}\right)^{2} \sum_{n=2,3} 2\left(v_{n}^{t}\right)^{2} \cos (n \Delta \phi) .
\end{aligned}
$$

One sees that, because of the change in the trigger angle $\phi_{s}(0 \rightarrow \pi / 2)$, the cosine dependence of the background contribution has an opposite sign, as compared to the in-plane correlation. We found the in-plane/out-of-plane effect! To illustrate these results, plots are shown in Fig. 3, with an appropriate choice of parameters. 


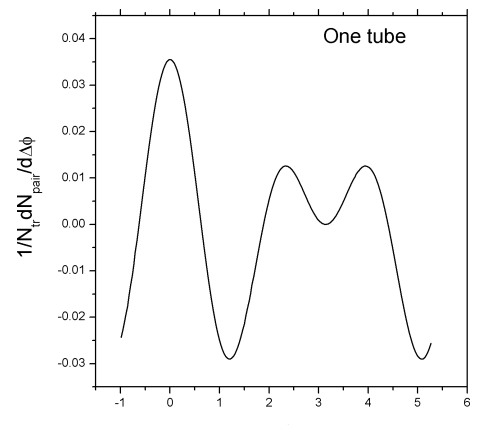

$\Delta \phi$

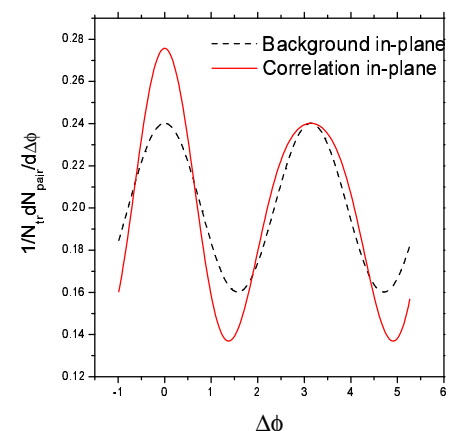

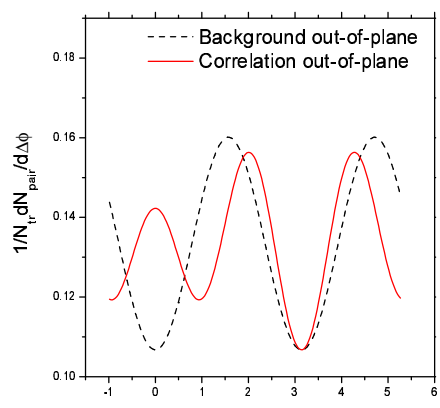

$\Delta \phi$

Figure 3: Plots of two-particle correlation. From the left to the right, (i) the one-tube contribution; (ii) the one from the background (dashed line) and the total one (solid line) for in-plane triggers, as given by Eq.(2.9); and (iii) the corresponding ones for the out-of-plane triggers Eq.(2.10).

\section{Conclusions}

We conclude that the NeXSPheRIO code gives correct qualitative behavior of the in-plane/outof-plane effect. A simplified analytical parametrization, based on one-tube model, shows that this effect appears because, besides the contribution coming from the peripheral tube, additional contribution arises from the background elliptical flow, due to the multiplicity fluctuation. The latter is back-to-back $(\Delta \phi=0, \pi)$ in the case of in-plane triggers $\left(\phi_{S} \sim 0\right)$ and rotated by $\pi / 2$ $(\Delta \phi=-\pi / 2, \pi / 2)$ in the case of out-of-plane triggers $\left(\phi_{S} \sim \pi / 2\right)$.

\section{Acknowledgements}

We acknowledge funding from FAPESP and CNPq.

\section{References}

[1] Yogiro Hama, Rone P.G. Andrade, Frédérique Grassi and Wei-Liang Qian, Proceedings of ISMD2011; arXiv:1111.6935 [hep-ph].

[2] PHENIX Collab., A. Adare et al., Phys. Rev. C 78 (2008), 014901.

[3] STAR Collab., B.I. Abelev et al., Phys. Rev. Lett. 102 (2009), 052302.

[4] PHOBOS Collab., B. Alver et al., Phys. Rev. C 81 (2010), 024904.

[5] ALICE Collab., K. Aamodt et al., Phys. Rev. Lett. 107 (2011), 032301.

[6] B. Wyslouch for the CMS Collab., arXiv:1107.2895.

[7] P. Steinberg for the ATLAS Collab., arXiv:1107.2182.

[8] J. Takahashi et al., Phys. Rev. Lett. 103 (2009), 242301.

[9] H.J. Drescher et al., Phys. Rev. C 65 (2002), 054902.

[10] C.E. Aguiar et al., J. Phys. G 27 (2001), 75. 
[11] Y. Hama et al., Nonlin. Phenom. Complex Sys. 12 (2010), 466.

[12] R.P.G. Andrade et al., J. Phys. G 37 (2010), 094043.

[13] R.P.G. Andrade et al., Nucl. Phys. A 854 (2011), 81.

[14] R.P.G. Andrade et al., arXiv:1012.5275 [hep/ph].

[15] Y. Hama et al., arXiv:1012.1342 [hep/ph].

[16] STAR Collab., A. Feng et al., J.Phys. G 35 (2008), 104082.

[17] STAR Collab., H. Agakishiev et al., arXiv:1010.0690 [nucl-ex].

[18] R. Andrade, F. Grassi, Y. Hama, W.-L. Qian, arXiv:1012.5275 [hep-ph]. 